\section{Statistische Erfassung}

Eine Erfassung der Verfahren häuslicher Gewalt bei der Staatsanwaltschaft kann, wenn sie in gesonderten Sachgruppen erfolgt, die Auswertung der Verfahren auch für politische und wissenschaftliche Zwecke erleichtern. Soweit bisher überhaupt eine statistische Erfassung erfolgt, geschieht dies auf der Basis der in einzelnen Bundesländern ${ }^{3}$ unterschiedlich vorgegebenen Definitionen lediglich für die Staatsanwaltschaften. Die Einführung gesonderter Sachgruppen in der Statistik für die Staatsanwaltschaften und Gerichte auf der Basis der oben genannten Definition würde eine Prüfung des Verfahrensausgangs bei den Staatsanwaltschaften ebenso wie bei den Ge- richten in erster Instanz ermöglichen, da die Sachgruppen insoweit einheitlich verwendet werden. Der Verfahrensverlauf und damit zumindest teilweise die Effektivität der Bekämpfung häuslicher Gewalt durch die Justiz würden dadurch im Längsschnitt überprüfbar. Weitere und neue Instrumente könnten auf dieser Basis entwickelt und evaluiert, ineffektive Maßnahmen im Bereich der Justiz reformiert werden.

3 Hessen, Mecklenburg-Vorpommern, Schleswig-Holstein; in Niedersachsen ist eine flächendeckende Erfassung parallel zu den zum 1.7.2010 flächendeckend einzurichtenden Sonderdezernaten der Staatsanwaltschaften vorgesehen.

\title{
Das Sorgerecht der nichtehelichen Väter
}

\section{Dr. Angelika Nake \\ Vorsitzende der Kommission Zivil-, Familien- und Erbrecht des djb; Rechtsanwältin, Darmstadt}

Mit Urteil vom 3. Dezember 2009 hat der Europäische Gerichtshof für Menschenrechte (EGMR) zu Art. 8 I, Art. 14 EMRK und $\$ 1626$ a II BGB entschieden, dass Deutschland die Väter nichtehelicher Kinder beim Zugang zur gemeinsamen Sorge diskriminiere. Das Gericht weist insoweit darauf hin, dass es keine objektive Rechtfertigung dafür gibt, dass das Sorgerecht des nichtehelichen Vaters einer gerichtlichen Einzelfallprüfung nicht zugänglich ist. ${ }^{1}$

Dem lag folgender Fall zugrunde: Der 1964 geborene Beschwerdeführer ist Vater eines außerhalb einer Ehe im Jahre 1995 geborenen Kindes. Die Eltern lebten seit 1993 zusammen und trennten sich im August 1998. Bis Januar 2001 wohnte das Kind bei dem Vater, die Mutter wohnte in einer anderen Wohnung, allerdings im gleichen Haus. Im Januar 2001 zog die Mutter dann mit dem Kind aus. Der Vater hatte mit dem Kind einen umfangreichen Umgang, er sah das Kind Mittwochnachmittag bis Donnerstagmorgen und Sonntag ab 10 Uhr bis Montagmorgen sowie die hälftigen Ferien. Insgesamt etwa vier Monate im Jahr. Die Mutter wollte die Sorgeerklärung nicht abgeben, so dass der Vater im Jahr 2001 die gemeinsame Sorge beim Familiengericht beantragte. Im Instanzenzug wurde der Antrag abgelehnt bzw. zur Verfassungsbeschwerde nicht angenommen.

Der Europäische Gerichtshof führt zur Verletzung des Art. 14 i.V.m. Art. 8 EMRK aus, dass eine Rechtfertigung für die Ungleichbehandlung nicht bestehe, anders als das BVerfG in seiner Grundsatzentscheidung vom 29. Januar 2003. ${ }^{2}$ Hier hat das BVerfG festgestellt, dass das Kindeswohl es verlange, dass das Kind ab seiner Geburt eine Person hat, die für es rechtsverbindlich handeln kann. Angesichts der Unterschiedlichkeit der Lebensverhältnisse, in die nichteheliche Kinder hineingeboren werden, sei es verfassungsgemäß, dass die Mut- ter die elterliche Sorge alleine habe. Die Mutter kann dann im Rahmen einer Sorgeerklärung die Mitsorge auf den Vater übertragen. Zum Schutz des Kindeswohls sei es aber gerechtfertigt, dass zunächst die Mutter das Sorgerecht erhält. Das Gericht akzeptierte auch, dass es gewichtige Gründe dafür geben kann, das Sorgerecht nicht jedem nichtehelichen Vater zu übertragen, zum Beispiel dann, wenn Streit oder mangelnde Kommunikation zwischen den Eltern dem Kindeswohl schaden würden.

Im vorliegenden Fall teilt der EGMR diese Bedenken nicht. Er führt aus, dass hier die Eltern gemeinsam gelebt haben, dreieinhalb Jahre in nichtehelicher Lebensgemeinschaft und weitere zwei Jahre noch nachher im gleichen Haus. Das Gericht weist darauf hin, dass dem Vater die Möglichkeit eingeräumt werden muss, gerichtlich überprüfen zu lassen, ob das gemeinsame Sorgerecht dem Kindeswohl dienlich ist. Es stellt anhand der Untersuchung der Bundesregierung fest, dass es nicht nur kindeswohlbezogene Erwägungen der Mütter wären, dem Vater das Mitsorgerecht nicht per Sorgeerklärung übertragen zu lassen. Keine Kindeswohlgefährdung sieht das Gericht in der seiner Ansicht nach notwendigen Klage zur Sorgerechtsübertragung, da diese auch im Rahmen von ehelichen Kindschaftsverhältnissen notwendig sein könne.

Dies ist soweit richtig; auch bei einer Scheidung oder Trennung kommen Anträge zur Übertragung der alleinigen Sorge auf den einen oder anderen Ehegatten vor. Das Sorgerecht wird in den Fällen auf ein Elternteil übertragen, in welchen die Weiterführung der gemeinsamen Sorge kindeswohlwidrig wäre. Das BVerfG legt als Prämisse zugrunde, dass die Ausübung der elterlichen Sorge im Sinne des Kindeswohls ein Mindestmaß an Einverständnis zwischen den Elternteilen voraussetzt. Wenn dieses Mindestmaß an Einverständnis nicht vorliegt, kann ein gemeinsames Sorgerecht kindeswohlför-

1 EGMR, FamRZ 2010, $103 \mathrm{ff}$.

2 NJW 2003, 955; FPR 2003, 205 = FamRZ 2003, 285 
dernd nicht ausgeübt werden. Zwar gibt beispielsweise der Verein Väter für Kinder ${ }^{3}$ zu bedenken, dass die Persönlichkeitsentwicklung des Kindes durch die Erfahrung gefördert werde, wie sich die Eltern in wichtigen Fragen streitig auseinandersetzen, um schließlich eine Einigung zu finden. Dies wird aber leider der Realität der meisten Differenzen zwischen Elternteilen nicht gerecht.

Der wesentliche Unterschied zwischen der Situation der Übertragung des Sorgerechts auf einen Elternteil im Nachgang zur Trennung und Scheidung und der Übertragung des gemeinsamen Sorgerechts auf den anderen Elternteil ist aber, dass mit der Beendigung der gemeinsamen Sorge und der Übertragung auf einen Elternteil zum Wohle des Kindes ein Streit, der sich negativ auf das Kindeswohl auswirkt, gerichtlich beendet wird. Jedenfalls in den Punkten, die von der Sorgerechtsübertragung betroffen werden. Anders stellt sich die rechtliche Situation dar, wenn das Mitsorgerecht auf den anderen Elternteil per Gericht übertragen wird. Die gemeinsame Sorge bedeutet, dass wesentliche Entscheidungen im Leben des Kindes von nun an einvernehmlich getroffen werden müssen. Erst jetzt aber zeichnet sich ab, ob überhaupt ein Konsens über die Erziehung, Bildung oder sogar den Namen des Kindes zwischen den Eltern erzielt werden kann. In der Regel ist die Situation nicht so - wie in dem Fall, den das EGMR zu entscheiden hatte -, dass sich der Vater schon mehrere Jahre - erst beim Zusammenleben und dann mit einem umfänglichen Umgang - „bewährt“ hat. In der Regel wird es so sein, dass der Antrag gestellt wird, wenn das Kind gerade geboren wird und sich im Laufe der Zeit erst entwickelt, ob überhaupt ein Konsens zwischen den Eltern möglich ist.

Der grundsätzlichen Zuordnung des Kindes zur Mutter liegt die biologische Tatsache zugrunde, dass die Mutter - im Gegensatz zum Vater - von Anfang an bekannt ist. Die Beziehung - rechtlich und faktisch - zwischen Mutter und Kind ist insoweit gesichert. Jedenfalls dann, wenn Prämisse für die Abstammung von der Mutter die Geburt des Kindes ist und nicht die biologische Abstammung. Statistisch gesehen werden 50 Prozent der Kinder unverheirateter Eltern in bestehende Lebensgemeinschaften hinein geboren. ${ }^{4}$ Das BVerfG hat mit seinem Urteil vom 29. Januar 2003 das Regelungskonzept der $\mathbb{S} \mathbb{S}$ 1626a, 1672 BGB im Wesentlichen für verfassungskonform erklärt. ${ }^{5}$ Insbesondere in Fällen, in denen die Eltern mit dem Kind zusammenlebten und beide ihre Kooperationsbereitschaft schon durch gemeinsame tatsächliche Sorge für das Kind zum Ausdruck gebracht hätten, habe der Gesetzgeber davon ausgehen dürfen, dass die Eltern die nunmehr bestehende gesetzliche Möglichkeit einer gemeinsamen Sorgetragung in der Regel nutzten und ihre tatsächliche Sorge durch Sorgeerklärungen auch rechtlich absicherten. ${ }^{6}$ Nur ausnahmsweise werde sich eine Mutter, die mit Vater und Kind zusammenlebt, dem Wunsch des Vaters nach einer gemeinsamen Sorge verweigern, wenn sie dafür schwerwiegende Gründe hat, die von der Wahrung des Kindeswohls getragen werden. ${ }^{7}$

Leider hat die vom BVerfG geforderte statistische Überprüfung der Sorgeerklärungen nicht dazu beigetragen, die Situa- tion zu beleuchten. Die Jugendämter haben zum Teil doppelte Speicherungen durchgeführt, so dass die Zahlen nicht aussagekräftig sind. Das Bundesjustizministerium hat auf seine Umfrage bei 440 Jugendämtern und 109 Rechtsanwälten bei einer Teilnahmequote von ca. 69,8 Prozent bei Jugendämtern und ca. 1,8 Prozent bei Rechtsanwälten ${ }^{8}$ zur Sorgeerklärung die folgenden Ergebnisse erhalten:

In Frage 2 wurden die Teilnehmer um eine Schätzung gebeten, wie viele nicht verheiratete Eltern zusammenleben oder längere Zeit zusammengelebt haben, ohne die gemeinsame Sorge zu begründen. Hierdurch sollte ein gewisser Eindruck entstehen, ob dies häufiger oder eher selten vorkommt. Insbesondere sollte überprüft werden, ob ein Rückschluss auf die gesetzgeberische Annahme möglich ist, wonach Mütter, die mit Vater und Kind zusammenleben, nur in Ausnahmefällen die Begründung der gemeinsamen Sorge ablehnen. Nach den Ergebnissen von Gesamtauswertung, Sonderauswertung und Kombinationsauswertung leben etwa 25 bis 75 Prozent aller Eltern zusammen oder haben zumindest längere Zeit zusammengelebt, ohne die gemeinsame Sorge zu begründen. Genauere Angaben sind nicht möglich, da die Antworten sehr unterschiedlich waren und auf bloßen Schätzungen beruhen. Insofern ist ein Rückschluss auf die Richtigkeit der oben genannten gesetzgeberischen Annahme nicht möglich.

Es wurde auch die Frage nach den Motiven der Mütter für die Ablehnung der gemeinsamen Sorge gestellt. Den Teilnehmern wurden verschiedene Motive zur Auswahl gestellt, die zum Teil kindeswohlorientiert (z. B. „häufig Konflikte der Eltern“) und zum Teil kindeswohlfern (z. B. „Mutter möchte sich am Vater rächen“) waren. Im Ergebnis wurden von den Teilnehmenden sowohl kindeswohlorientierte Gründe als auch kindeswohlferne Gründe genannt.

Die Ergebnisse der Befragung und die weiteren von den Teilnehmenden genannten Motive zeigen, dass die Gründe für die Ablehnung der gemeinsamen Sorge sehr vielschichtig sind. Sie deuten darauf hin, dass die Entscheidung gegen die gemeinsame Sorge häufig auch emotional gesteuert ist und dabei u. a. Verlustängste, Besitzansprüche oder Kontrollbedürfnisse oder auch der Einfluss dritter Personen eine Rolle spielen können. Diese Ergebnisse sind jedoch mit äußerster Vorsicht zu interpretieren. Die Angaben der Befragten beruhen in aller Regel ausschließlich auf Informationen, die diese von den beratenen Vätern erhalten haben, und konnten von den Teilnehmenden nicht verifiziert werden. Viele Teilnehmende der Umfrage haben besonders darauf hingewiesen, dass ein Kontakt mit den Müttern nicht bestand. Es ist daher möglich, dass der

3 〈http://www.vaeterfuerkinder.de> (Zugriff 21.4.2010).

4 BT-Drs. 16/6078 vom 11.7.2007, <http://www.dnoti.de/DOC/2007/ BT_Drs_16_6078.pdf> (Zugriff 21.4.2010)

5 BVerfG, Urteil vom 29.1.2003-1 BvL 20/99, 1 BvR 933/o1, BVerfGE $107,150 \mathrm{ff}$.

6 BVerfGE 107, 150 ff., 176.

7 BVerfGE 107, 177.

8 <http://www.vaeter-aktuell.de/Umfrage_BMJ_1626a_Zusammenfassung.pdf> (Zugriff 21.4.2010). 
- hier wiedergegebene - Eindruck der Beratungsperson mit den tatsächlichen Motiven der Mütter nicht übereinstimmt.

Im Ergebnis hat die vom Bundesministerium der Justiz durchgeführte Befragung von Jugendämtern und Rechtsanwält(inn)en ein sehr vielschichtiges Bild ergeben. Die Angaben der Teilnehmer(innen) beruhen ausschließlich auf Erinnerungswerten und Schätzwerten, nicht auf belastbaren statistischen Angaben.

Es ergibt sich hieraus, dass insbesondere die Einschätzung des EGMR, dass nicht immer kindbezogene Gründe der Anlass dafür sind, die Sorgeerklärung nicht abzugeben, insbesondere auf der Sicht der Väter beruhen und auf der persönlichen Einschätzung der Jugendamtsmitarbeiter(innen) und der teilnehmenden Rechtsanwält(inn)e(n). Eine echte Auseinandersetzung mit den Motiven hat nicht stattgefunden.

Auch der internationale Vergleich, der immer bemüht wird, führt hier nicht weiter. Die Antwort der Bundesregierung auf eine Anfrage der Grünen gibt an, dass die überwiegende Zahl der Rechtsordnungen in den Mitgliedstaaten der Europäischen Union den Eltern das Sorgerecht gemeinsam zuordne, und zwar kraft Gesetzes und unabhängig vom Personenstand der Eltern. ${ }^{9}$

Wie bereits der Richter am EGMR Schmitt in seinem Sondervotum zur Entscheidung richtig ausführt, gibt es einen europäischen Konsens zu der Frage nicht. Richtig ist, dass eine Vielzahl von europäischen Staaten die Entscheidung trifft, dass die elterliche Mitsorge des nichtehelichen Vaters entweder automatisch mit der Geburt - bzw. der Feststellung der Vaterschaft - entsteht und ein anderer Teil die Entscheidung trifft, dass der nichteheliche Vater jedenfalls per Antrag das Sorgerecht übertragen erhalten kann. So kennen beispielsweise Belgien, Bulgarien, Belarus, Tschechien, Ungarn, Polen, Russland, Slowenien, die Slowakei und die Ukraine die Mitsorge des nichtehelichen Vaters. Andere europäische Länder lassen die Mitsorge auf Antrag zu, wobei es auch hier gravierende Unterschiede gibt. Frankreich lässt den Antrag des nichtehelichen Vaters ausreichen, wenn er innerhalb des ersten Jahres nach der Geburt des Kindes gestellt wird. Andere Länder überprüfen die Situation der Eltern und des Kindes per Gericht, wenn die Mutter nicht zustimmt. Hier von einem europäischen Konsens zu sprechen, ginge sicher zu weit.

Im Gegensatz zu der Beendigung der Mitsorge bei ehelichen Kindern ist die Situation des nichtehelichen Vaters, der die Mitsorge begehrt, in der Regel völlig anders. Auch der Fall, den das EGMR zu entscheiden hatte, stellt nicht den Regelfall dar. Bei dem beantragenden Vater kann es sich auch um einen Vater handeln, der sich eben noch nicht als zuverlässiger Partner in Fragen um das Kind bewährt hat. Hier werden Väter zu finden sein, die keinen Unterhalt für das Kind zahlen, teilweise über Jahre hinweg, und die das Umgangsrecht mit dem Kind nicht regelmäßig wahrnehmen und trotzdem die rechtliche Verantwortung für das Kind mit übernehmen wollen. Hier werden Eltern aufeinandertreffen, die keinen Grundkonsens in Fragen der Erziehung haben. Eltern, die sich kaum kennen. Das Kind hat jedenfalls in jeder Situation einen Anspruch darauf, dass seine Eltern die für seine Zukunft wichtigen Fragen entscheiden. Sind die Eltern hierzu nicht in der Lage, wird diese Entscheidung nötigenfalls mit gerichtlicher Hilfe getroffen werden müssen. Gerichte werden dann gegebenenfalls in noch weiterem Umfang als heute entscheiden müssen, welcher Kindergarten für das Kind der richtige ist und welchen Namen das Kind tragen soll. Wenn aber das Gericht eine Entscheidung trifft, die dem Wunsch desjenigen widerspricht, bei dem das Kind lebt, muss dieser dann die Entscheidung des Gerichts tragen, weiter entwickeln und in der Zukunft befördern.

Ob dies der täglichen Realität in Familien entspricht und insbesondere dem Kindeswohl dienlich ist, wird die Frage sein, die der Gesetzgeber nun zu beantworten hat.

9 BT-Drs. 16/6078 vom 11.7.2007, <http://www.dnoti.de/DOC/2007/ BT_Drs_16_6078.pdf> (Zugriff 21.4.2010).

\section{Die gleichgeschlechtliche Lebenspartnerschaft - Sorgerecht und Adoption}

\author{
Daniela Helm \\ Rechtsreferendarin, Berlin
}

\section{Dr. Kerstin Niethammer-Jürgens}

Mitglied der Kommission Zivil-, Familien- und Erbrecht des djb; Rechtsanwältin, Potsdam

\section{Das Sorgerecht}

Das gemeinsame Sorgerecht der Eltern besteht auch nach Trennung und Aufnahme einer gleichgeschlechtlichen Le- benspartnerschaft fort. Die Homosexualität darf bei der Sorgerechtsentscheidung keine Rolle spielen, insbesondere darf sie nicht als negativ gewertet werden.

\section{Das „kleine Sorgerecht“}

Steht die elterliche Sorge beiden Elternteilen gemeinsam zu, zum Beispiel aufgrund einer zuvor bestandenen Ehe, wie es heute nach einer Scheidung der Regelfall ist, ist der (die) eingetragene Lebenspartner(in) nicht zur Ausübung des „kleinen Sorgerechts“ im Sinne von $\mathbb{\$} 9$ Abs. 1 LPartG befugt. Ist das 